\title{
The role of positive boundary data in generalized clamped plate equations
}

\author{
Hans-Christoph Grunau \\ Fachgruppe Mathematik \\ Universität Bayreuth \\ D-95440 Bayreuth \\ Germany
}

\author{
Guido Sweers \\ Vakgroep Algemene Wiskunde \\ Postbus 5031 \\ Technische Universiteit Delft \\ NL-2600 GA Delft \\ The Netherlands
}

\begin{abstract}
Positivity phenomena in higher order elliptic Dirichlet problems as e.g. in the clamped plate equation are in general rather subtle. It depends on the domain and on the particular form of the operator whether there are comparison principles or not. Until now most papers concentrated on positivity with respect to the right-hand side, i.e. on positivity of the Green function itself. In the present paper we focus on the role of the boundary data, i.e. on positivity of certain Poisson kernels. While it is expected that the Poisson kernel of highest order behaves similarly as the Green function, it may be surprising that for Dirichlet problems of arbitrary order and in any dimension there is also a positivity result with respect to a second Poisson kernel. Furthermore a perturbation theory for this result is developed.
\end{abstract}

Keywords: Clamped plate equation, positivity, comparison principle, Poisson kernel.

AMS-Classification: 35J40,35B50.

\section{Introduction}

Like Boggio [Bo1] and Hadamard [Ha] (1901/08) one might conjecture that positive data $f \geq 0, \varphi \geq 0, \psi \geq 0$ in the Dirichlet problem

(1) $\left\{\begin{array}{l}(-\Delta)^{2} u=f \text { in } \Omega, \\ u\left|\partial \Omega=\psi, \quad\left(-\frac{\partial u}{\partial \nu}\right)\right| \partial \Omega=\varphi,\end{array}\right.$ 
yield positive solutions $u \geq 0$. Here $\Omega \subset \mathbb{R}^{n}$ is a smoothly bounded domain and $\nu$ is the exterior unit normal at $\partial \Omega$. In two dimensions (1) is the clamped plate equation. Here $\Omega$ is the shape of the plate, $f$ is the perpendicular load, the boundary data $\varphi$ and $\psi$ describe the "clamping" and $u$ is the deflection of the plate.

Most authors concentrated on the Green function $G_{2, n, \Omega}$ for the Dirichlet problem (1) in case of homogeneous boundary data $\varphi=\psi=0$. In $G_{2, n, \Omega}$ the 2 stands for biharmonic and $n$ for the dimension. Boggio [Bo2] could show by explicit calculation that if $\Omega=B$ is the (unit) ball the Green function $G_{m, n}:=G_{m, n, B}$ for any power $(-\Delta)^{m}$ with Dirichlet boundary conditions is positive. The case that $\Omega=B$ turned out to be a special case. Numerous counterexamples (see Duffin [Du], Garabedian [Ga], Coffman and Duffin [CD], Shapiro and Tegmark [ST] and many others) have shown that this result actually does not hold in arbitrary domains $\Omega$.

A perturbation theory for Boggio's positivity result has been developed by the authors in [GS1] with respect to lower order terms of the differential operator and in [GS2] with respect to the domain and the highest order terms of the differential operator in two dimensions.

In the present note we focus on the role of the boundary data $\varphi$ and $\psi$ in the case that the domain $\Omega$ is the (unit) ball $B$. Consequently we may assume that $f=0$. As we pointed out in [GS3], cf. also [He], if $\psi=0$ the positivity behaviour of the Dirichlet problem (1) with respect to the highest order boundary datum $\varphi$ is more or less the same as with respect to the right-hand side. But if also $\psi \geq 0$, $\psi \not \equiv 0$ is considered the situation becomes more involved. In the unit ball $B$ we have the following explicit formula for the solution $u$ of (1) (see [Ni, p. 34]):

(2) $u(x)=\int_{\partial B} K_{2, n}(x, y) \psi(y) d \omega(y)+\int_{\partial B} L_{2, n}(x, y) \varphi(y) d \omega(y), \quad x \in B$,

where

(3) $\quad K_{2, n}(x, y)=\frac{1}{2 \omega_{n}} \frac{\left(1-|x|^{2}\right)^{2}}{|x-y|^{n+2}}\left\{2+(n-4) x \cdot y-(n-2)|x|^{2}\right\}$,

(4) $L_{2, n}(x, y)=\frac{1}{2 \omega_{n}} \frac{\left(1-|x|^{2}\right)^{2}}{|x-y|^{n}}$,

with $x \in B, y \in \partial B ; \omega_{n}$ denotes the $(n-1)$-dimensional surface area of the unit ball. Evidently $L_{2, n}>0$ for any $n$, while $K_{2, n}>0$ only for $n \leq 4$ and $K_{2, n}$ changes sign for $n \geq 5$.

In the next section we will show that if $\Omega=B$ the Dirichlet problem (1) may be reformulated in such a way that we have a positivity result with respect to both boundary data in any dimension. Moreover for $n \leq 3$ and in particular for $n=2$ the above mentioned result may be sharpened so that if $\psi\left(x_{0}\right)>0$ for some $x_{0} \in \partial B$, also negative values for $\varphi$ near $x_{0}$ are admissible.

In the last section we switch to polyharmonic Dirichlet problems of arbitrary order $2 \mathrm{~m}$. We will show, allowing some "small" lower order perturbations of the 
differential operator, positivity with respect to the Dirichlet data of order $(m-1)$ and $(m-2)$ in any dimension $n$, provided the other boundary data are prescribed homogeneously and the positivity assumption is posed in a suitable way. In [GS3] we could only show a perturbation result for $n \leq 3, m=2$.

\section{The appropriate positivity assumption for the clamped plate equation}

In order to find the adequate positivity assumption on the boundary data in the Dirichlet problem

(5) $\left\{\begin{array}{l}(-\Delta)^{2} u=0 \text { in } B \\ u\left|\partial B=\psi, \quad\left(-\frac{\partial u}{\partial \nu}\right)\right| \partial B=\varphi\end{array}\right.$

the key observation is that the negative part of the kernel $K_{2, n}$ corresponding to $\psi$ has the same form as the kernel $L_{2, n}$ corresponding to the datum $\varphi$.

Lemma 2.1. Let $s \in \mathbb{R}, s \geq \frac{1}{2}(n-4)$. Then for

(6) $\hat{K}_{2, n, s}(x, y):=K_{2, n}(x, y)+s L_{2, n}(x, y), \quad x \in B, \quad y \in \partial B$,

we have

$$
\hat{K}_{2, n, s}(x, y)>0
$$

Proof. We observe that for $x \in B, y \in \partial B$ (i.e. $|y|=1$ ) we have

$$
\begin{aligned}
K_{2, n}(x, y) & =\frac{1}{2 \omega_{n}} \frac{\left(1-|x|^{2}\right)^{2}}{|x-y|^{n+2}}\left\{\frac{n}{2}\left(1-|x|^{2}\right)-\frac{1}{2}(n-4)|x-y|^{2}\right\} \\
& =\frac{n}{4 \omega_{n}} \frac{\left(1-|x|^{2}\right)^{3}}{|x-y|^{n+2}}-\frac{1}{2}(n-4) L_{2, n}(x, y) .
\end{aligned}
$$

Proposition 2.2. Let $\varphi \in C^{0}(\partial B), \psi \in C^{1}(\partial B)$ and $s \geq \frac{1}{2}(n-4)$. If we assume that

$$
\psi(x) \geq 0 \quad \text { and } \quad \varphi(x) \geq s \psi(x) \text { for } x \in \partial B,
$$

then the uniquely determined solution $u \in C^{4}(B) \cap C^{1}(\bar{B})$ of the Dirichlet problem (5) is positive:

$$
u \geq 0 \quad \text { in } B \text {. }
$$


Proof. From (2) and (6) we obtain:

$$
\begin{aligned}
u(x) & =\int_{\partial B} K_{2, n}(x, y) \psi(y) d \omega(y)+\int_{\partial B} L_{2, n}(x, y) \varphi(y) d \omega(y) \\
& =\int_{\partial B} \hat{K}_{2, n, s}(x, y) \psi(y) d \omega(y)+\int_{\partial B} L_{2, n}(x, y)(\varphi(y)-s \psi(y)) d \omega(y)
\end{aligned}
$$

Remarks. 1) For $n=1,2,3$, also negative values for $s$ are admissible.

2) On $B_{R}:=\left\{x \in \mathbb{R}^{n}:|x|<R\right\}$ the condition is $s \geq \frac{1}{2 R}(n-4)$.

We are interested in whether this positivity result remains under perturbations of the prototype problem (5). Since in higher order Dirichlet problems quite similar phenomena can be observed, we develop the perturbation theory for the biharmonic Dirichlet problem (5) as a special case of the perturbation theory for the polyharmonic Dirichlet problem (7) below. The latter is subject of the following section.

\section{Higher order equations. Perturbations}

In what follows we always assume $m \geq 2$.

First we consider the polyharmonic prototype Dirichlet problem:

(7)

$$
\begin{cases}(-\Delta)^{m} u=0 & \text { in } B \\ \left(-\frac{\partial}{\partial \nu}\right)^{j} u=0 & \text { on } \partial B \text { for } j=0, \ldots, m-3 \\ \left(-\frac{\partial}{\partial \nu}\right)^{m-2} u=\psi & \text { on } \partial B \\ \left(-\frac{\partial}{\partial \nu}\right)^{m-1} u=\varphi & \text { on } \partial B\end{cases}
$$

No uniform positivity result can be expected with respect to the boundary data of order $0, \ldots, m-3$, as we will explain below in the example following Proposition 3.2. So we prescribe these data homogeneously. Such behaviour is in contrast with the radially symmetric case $u=u(|x|)$ (cf. Soranzo [So, Proposition 1, Remark 9]). For a different way of formulating the Dirichlet problem involving powers of the Laplacian for the boundary data see the remark at the end of this section.

After some elementary calculations we find from [Ed] or directly from (2) that for $\varphi \in C^{0}(\partial B), \psi \in C^{1}(\partial B)$ the solution $u \in C^{2 m}(B) \cap C^{m-1}(\bar{B})$ to the Dirichlet problem (7) is given by

$$
u(x)=\int_{\partial B} K_{m, n}(x, y) \psi(y) d \omega(y)+\int_{\partial B} L_{m, n}(x, y) \varphi(y) d \omega(y), \quad x \in B,
$$


where

$$
\begin{aligned}
K_{m, n}(x, y)= & \frac{1}{2^{m}(m-2) ! \omega_{n}} \frac{\left(1-|x|^{2}\right)^{m}}{|x-y|^{n+2}} \\
& \cdot\left\{n\left(1-|x|^{2}\right)-(n-2-m)|x-y|^{2}\right\}, \\
\text { (10) } L_{m, n}(x, y)= & \frac{1}{2^{m-1}(m-1) ! \omega_{n}} \frac{\left(1-|x|^{2}\right)^{m}}{|x-y|^{n}},
\end{aligned}
$$

with $x \in B, y \in \partial B$. Like in Section 2 we have the following results.

Lemma 3.1. Let $s \in \mathbb{R}$ satisfy $s \geq \frac{1}{2}(n-2-m)(m-1)$. Then for

(11) $\hat{K}_{m, n, s}(x, y):=K_{m, n}(x, y)+s L_{m, n}(x, y), \quad x \in B, \quad y \in \partial B$, we have

$$
\hat{K}_{m, n, s}(x, y)>0 .
$$

Proof.

$$
\begin{aligned}
\hat{K}_{m, n, s}(x, y)= & \frac{1}{2^{m}(m-2) ! \omega_{n}} \frac{\left(1-|x|^{2}\right)^{m}}{|x-y|^{n+2}} \\
& \cdot\left\{n\left(1-|x|^{2}\right)+\left(\frac{2 s}{m-1}-(n-2-m)\right)|x-y|^{2}\right\} .
\end{aligned}
$$

Proposition 3.2. Let $\varphi \in C^{0}(\partial B), \psi \in C^{1}(\partial B)$ and $s \geq \frac{1}{2}(n-2-m)(m-1)$. If

$$
\psi(x) \geq 0 \quad \text { and } \quad \varphi(x) \geq s \psi(x) \quad \text { for } x \in \partial B,
$$

then the uniquely determined solution $u \in C^{2 m}(B) \cap C^{m-1}(\bar{B})$ of the Dirichlet problem (7) is positive:

$$
u \geq 0 \text { in } B \text {. }
$$

Remark. On $B_{R}=\left\{x \in \mathbb{R}^{n}:|x|<R\right\}$ the condition is $s \geq \frac{1}{2 R}(n-2-m)(m-1)$.

Example. In the triharmonic Dirichlet problem

$$
\begin{cases}(-\Delta)^{3} u=0 & \text { in } B, \\ u=\chi & \text { on } \partial B, \\ \left(-\frac{\partial}{\partial \nu}\right) u=\psi & \text { on } \partial B, \\ \left(\frac{\partial}{\partial \nu}\right)^{2} u=\varphi & \text { on } \partial B,\end{cases}
$$


the solution is given by

$$
\begin{aligned}
u(x)= & \int_{\partial B} H_{3, n}(x, y) \chi(y) d \omega(y)+\int_{\partial B} K_{3, n}(x, y) \psi(y) d \omega(y) \\
& +\int_{\partial B} L_{3, n}(x, y) \varphi(y) d \omega(y), \quad x \in B
\end{aligned}
$$

where $K_{3, n}$ and $L_{3, n}$ are defined above and

$$
\begin{aligned}
H_{3, n}(x, y)= & \frac{1}{16 \omega_{n}} \frac{\left(1-|x|^{2}\right)^{3}}{|x-y|^{n+4}} \\
& \cdot\left\{n(n+2)\left(1-|x|^{2}\right)^{2}+(n-4)(n-8)|x-y|^{4}\right. \\
& \left.\quad-2 n(n-7)\left(1-|x|^{2}\right)|x-y|^{2}-4 n|x-y|^{2}\right\}
\end{aligned}
$$

with $x \in B, y \in \partial B$. For any $n, x \rightarrow y, x$ "very close" to the boundary, $H_{3, n}$ takes on also negative values. By adding multiples of $L_{3, n}$ and $K_{3, n}$, only the terms $|x-y|^{4}$ and $\left(1-|x|^{2}\right)|x-y|^{2}$ in the curved brackets could be effected. In any case the most dangerous term $-4 n|x-y|^{2}$ remains.

As a starting point for the perturbation theory of Proposition 3.2 we describe the essential properties ot the integral kernels $\hat{K}_{m, n, s}$ and $L_{m, n}$.

For brevity we introduce for the distance to the boundary

(13) $d(x):=1-|x|, \quad x \in B$,

and for $f, g: M \subset \mathbb{R}^{k} \rightarrow \mathbb{R}^{+}$we will use the notation:

$$
\begin{aligned}
& f \sim g \text { on } M \quad \Leftrightarrow \quad \exists C>0 \forall x \in M: \frac{1}{C} f(x) \leq g(x) \leq C f(x), \\
& f \preceq g \text { on } M \quad \Leftrightarrow \quad \exists C>0 \forall x \in M: f(x) \leq C g(x) .
\end{aligned}
$$

Lemma 3.3. a) Let $s \geq \frac{1}{2}(n-2-m)(m-1)$. On $B \times \partial B$ (i.e. for $x \in B, y \in \partial B$ ) we have

(14) $\hat{K}_{m, n, s}(x, y)\left\{\begin{array}{l}\preceq|x-y|^{-n-1} d(x)^{m}, \\ \succeq|x-y|^{-n-2} d(x)^{m+1},\end{array}\right.$

(15) $L_{m, n}(x, y) \sim|x-y|^{-n} d(x)^{m}$.

b) If we assume additionally that $s>\frac{1}{2}(n-2-m)(m-1)$ then we have on $B \times \partial B$ :

(16) $\hat{K}_{m, n, s}(x, y)\left\{\begin{array}{l}\preceq|x-y|^{-n-1} d(x)^{m}, \\ \succeq|x-y|^{-n} d(x)^{m} .\end{array}\right.$ 
Proof. The claim follows from $1-|x|^{2} \sim d(x), d(x) \leq|x-y|$ and the expression in (12).

Remarks. 1) The estimation constants in (16) depend strongly on $s$.

2) If $s=\frac{1}{2}(n-2-m)(m-1)$ then we have $\hat{K}_{m, n, s}(x, y) \sim|x-y|^{-n-2} d(x)^{m+1}$, i.e. for $x \rightarrow \partial B \backslash\{y\}$ we have a zero of order $(m+1)$. We would have expected, and actually need in order to prove perturbation results, a zero of order $m$. Consequently in what follows we have to assume $s>\frac{1}{2}(n-2-m)(m-1)$. The estimate (16) is more appropriate. But as $\hat{K}_{m, n, s}(x, y) \nsim|x-y|^{-n-1} d(x)^{m}$ our perturbation result Theorem 3.5 below is (necessarily?) less general than the corresponding results in [GS1] and [GS2]. In particular we are not (yet?) able to consider domain perturbations.

For our purposes the following "3-G-type" estimates are essential. We use the multi-index notation $D^{\alpha}=\prod_{i=1}^{n}\left(\frac{\partial}{\partial x_{i}}\right)^{\alpha_{i}}$ for $\alpha \in \mathbb{N}_{0}^{n} ;|\alpha|=\sum_{i=1}^{n} \alpha_{i}$. We recall that $G_{m, n}=G_{m, n, B}$ denotes the Dirichlet Green function for $(-\Delta)^{m}$ in the unit ball $B \subset \mathbb{R}^{n}$.

Lemma 3.4. Let $s>\frac{1}{2}(n-2-m)(m-1), \alpha \in \mathbb{N}_{0}^{n}$. Then on $B \times \partial B \times B$ (i.e. for $x \in B, y \in \partial B, z \in B$ ) we have the following.

$$
\begin{aligned}
\frac{\left|D_{z}^{\alpha} G_{m, n}(x, z)\right| \hat{K}_{m, n, s}(z, y)}{\hat{K}_{m, n, s}(x, y)} & \preceq \\
& \preceq\left\{\begin{array}{r}
1, \quad \text { if }|\alpha|<2 m-n, \\
|x-z|^{2 m-1-n-|\alpha|}+|y-z|^{2 m-1-n-|\alpha|}, \\
\text { if }|\alpha| \geq 2 m-n .
\end{array}\right.
\end{aligned}
$$

$$
\frac{\left|D_{z}^{\alpha} G_{m, n}(x, z)\right| L_{m, n}(z, y)}{L_{m, n}(x, y)} \preceq
$$

$$
\preceq\left\{\begin{array}{l}
1, \quad \text { if }|\alpha| \leq 2 m-n \text { and } n \text { odd, } \\
\text { or if }|\alpha|<2 m-n, \\
\log \left(\frac{3}{|x-z|}\right), \quad \text { if }|\alpha|=2 m-n \text { and } n \text { even }, \\
|x-z|^{2 m-n-|\alpha|}+|y-z|^{2 m-n-|\alpha|}, \\
\text { if }|\alpha|>2 m-n .
\end{array}\right.
$$

Proof. We repeatedly employ $d(x) \leq|x-y|(y \in \partial B)$ without mentioning.

a) Proof of (17): We use estimate (16) of Lemma 3.3. 
The case: $|\alpha| \leq 2 m-n$ and $n$ odd, or $|\alpha|<2 m-n$.

Here we use Corollary A.3.

$$
\begin{aligned}
& \frac{\left|D_{z}^{\alpha} G_{m, n}(x, z)\right| \hat{K}_{m, n, s}(z, y)}{\hat{K}_{m, n, s}(x, y)} \\
& \preceq \quad \frac{d(x)^{m-\frac{n}{2}} d(z)^{m-\frac{n}{2}-|\alpha|} \min \left\{1, \frac{d(x)^{n / 2} d(z)^{n / 2}}{|x-z|^{n}}\right\} \frac{d(z)^{m}}{|z-y|^{n+1}}}{\frac{d(x)^{m}}{|x-y|^{n}}} \\
& \preceq \quad d(x)^{-\frac{n}{2}} d(z)^{2 m-\frac{n}{2}-|\alpha|} \min \left\{1, \frac{d(x)^{\frac{n}{2}} d(z)^{\frac{n}{2}}}{|x-z|^{n}}\right\} \\
& \quad \cdot|y-z|^{-n-1}\left(|x-z|^{n}+|y-z|^{n}\right) \\
& \quad d(z)^{2 m-|\alpha|}|y-z|^{-n-1}+d(x)^{-\frac{n}{2}} d(z)^{2 m-\frac{n}{2}-|\alpha|}\left(\frac{d(x)}{d(z)}\right)^{\frac{n}{2}}|y-z|^{-1} \\
& =d(z)^{2 m-|\alpha|}|y-z|^{-n-1}+d(z)^{2 m-n-|\alpha|}|y-z|^{-1} \preceq|y-z|^{2 m-1-n-|\alpha|} .
\end{aligned}
$$

The case: $|\alpha|=2 m-n$ and $n$ even.

We use Lemma A.2.

$$
\begin{aligned}
& \frac{\left|D_{z}^{\alpha} G_{m, n}(x, z)\right| \hat{K}_{m, n, s}(z, y)}{\hat{K}_{m, n, s}(x, y)} \\
& \preceq \frac{\log \left(2+\frac{d(x)}{|x-z|}\right) \min \left\{1, \frac{d(x)}{|x-z|}\right\}^{m} \min \left\{1, \frac{d(z)}{|x-z|}\right\}^{\max \{m-|\alpha|, 0\}} \frac{d(z)^{m}}{|z-y|^{n+1}}}{\frac{d(x)^{m}}{|x-y|^{n}}} \\
& \preceq \quad\left(1+\frac{d(x)}{|x-z|}\right) d(x)^{-m} d(z)^{m} \min \left\{1, \frac{d(x)}{|x-z|}\right\}^{m} \\
& \cdot \min \left\{1, \frac{d(z)}{|x-z|}\right\}^{\max \{m-|\alpha|, 0\}}|y-z|^{-n-1}\left(|x-z|^{n}+|y-z|^{n}\right) \\
& \quad\left\{d(x)^{-m} d(z)^{m}|y-z|^{-n-1}|x-z|^{n}+d(x)^{-m} d(z)^{m}|y-z|^{-1}\right. \\
& \left.+d(x)^{1-m} d(z)^{m}|y-z|^{-1}|x-z|^{-1}\right\} \\
& \cdot \min \left\{1, \frac{d(x)}{|x-z|}\right\}^{m} \min \left\{1, \frac{d(z)}{|x-z|}\right\}^{\max \{m-|\alpha|, 0\}} \\
& \qquad\left(\frac{d(x)}{d(z)}\right)^{\max \{m-n, 0\}}\left(\frac{d(z)}{|x-z|}\right)^{\max \{m-|\alpha|, 0\}} \\
& d(x)^{-m} d(z)^{m}|y-z|^{-n-1}|x-z|^{n}\left(\frac{d(x)}{|x-z|}\right)^{\min \{m, n\}}
\end{aligned}
$$




$$
\begin{aligned}
& +d(x)^{-m} d(z)^{m}|y-z|^{-1}\left(\frac{d(x)}{d(z)}\right)^{m} \\
& +d(x)^{1-m} d(z)^{m}|y-z|^{-n-1}|x-z|^{n-1}\left(\frac{d(x)}{|x-z|}\right)^{\min \{m, n\}-1} \\
& \cdot\left(\frac{d(x)}{d(z)}\right)^{\max \{m-n, 0\}}\left(\frac{d(z)}{|x-z|}\right)^{\max \{m-|\alpha|, 0\}} \\
& \quad+d(x)^{1-m} d(z)^{m}|y-z|^{-1}|x-z|^{-1}\left(\frac{d(x)}{d(z)}\right)^{m-1} \\
& \preceq \quad d(z)^{n}|y-z|^{-n-1}+|y-z|^{-1}+d(z)|y-z|^{-1}|x-z|^{-1} \\
& \qquad|x-z|^{-1}+|y-z|^{-1} .
\end{aligned}
$$

The case: $|\alpha|>2 m-n$ and $|\alpha| \leq m$.

We use Lemma A.2.

$$
\begin{aligned}
& \frac{\left|D_{z}^{\alpha} G_{m, n}(x, z)\right| \hat{K}_{m, n, s}(z, y)}{\hat{K}_{m, n, s}(x, y)} \\
& \preceq \quad \frac{|x-z|^{2 m-n-|\alpha|} \min \left\{1, \frac{d(x)}{|x-z|}\right\}^{m} \min \left\{1, \frac{d(z)}{|x-z|}\right\}^{m-|\alpha|} \frac{d(z)^{m}}{|z-y|^{n+1}}}{\frac{d\left(x-\left.y\right|^{m}\right.}{\mid m}} \\
& \preceq \quad d(x)^{-m} d(z)^{m}|x-z|^{2 m-n-|\alpha|}|y-z|^{-n-1} \\
& \quad \cdot \min \left\{1, \frac{d(x)}{|x-z|}\right\}^{m} \min \left\{1, \frac{d(z)}{|x-z|}\right\}^{m-|\alpha|}\left(|x-z|^{n}+|y-z|^{n}\right) \\
& \preceq \quad d(x)^{-m} d(z)^{m}|x-z|^{2 m-|\alpha|}|y-z|^{-n-1}\left(\frac{d(x)}{|x-z|}\right)^{m}\left(\frac{d(z)}{|x-z|}\right)^{m-|\alpha|} \\
& \quad+d(x)^{-m} d(z)^{m}|x-z|^{2 m-n-|\alpha|}|y-z|^{-1}\left(\frac{d(x)}{d(z)}\right)^{m} \\
& \preceq \quad d(z)^{2 m-|\alpha|}|y-z|^{-n-1}+|x-z|^{2 m-n-|\alpha|}|y-z|^{-1} \\
& \preceq \quad|x-z|^{2 m-1-n-|\alpha|}+|y-z|^{2 m-1-n-|\alpha|} .
\end{aligned}
$$

The case: $|\alpha|>2 m-n$ and $|\alpha|>m$.

$$
\begin{aligned}
& \frac{\left|D_{z}^{\alpha} G_{m, n}(x, z)\right| \hat{K}_{m, n, s}(z, y)}{\hat{K}_{m, n, s}(x, y)} \\
& \quad \preceq \frac{|x-z|^{2 m-n-|\alpha|} \min \left\{1, \frac{d(x)}{|x-z|}\right\}^{m} \frac{d(z)^{m}}{|z-y|^{n+1}}}{\frac{d(x)^{m}}{|x-y|^{n}}} \\
& \preceq d(x)^{-m} d(z)^{m}|x-z|^{2 m-n-|\alpha|}|y-z|^{-n-1}
\end{aligned}
$$




$$
\begin{aligned}
& \cdot \min \left\{1, \frac{d(x)}{|x-z|}\right\}^{m}\left(|x-z|^{n}+|y-z|^{n}\right) \\
\preceq \quad & d(x)^{-m} d(z)^{m}|x-z|^{2 m-|\alpha|}|y-z|^{-n-1} \\
& \cdot\left(\frac{d(x)}{|x-z|}\right)^{\min \{m, n\}}\left(\frac{d(x)}{d(z)}\right)^{\max \{m-n, 0\}} \\
& +d(x)^{-m} d(z)^{m}|x-z|^{2 m-n-|\alpha|}|y-z|^{-1}\left(\frac{d(x)}{d(z)}\right)^{m} \\
\preceq & d(z)^{\min \{m, n\}}|x-z|^{m-|\alpha|+\max \{m-n, 0\}}|y-z|^{-n-1} \\
& +|x-z|^{2 m-n-|\alpha|}|y-z|^{-1} \\
\preceq \quad & |x-z|^{m-|\alpha|+\max \{m-n, 0\}}|y-z|^{\min \{m-n, 0\}-1} \\
\preceq & +|x-z|^{2 m-n-|\alpha|}|y-z|^{-1} \\
& |x-z|^{2 m-1-n-|\alpha|}+|y-z|^{2 m-1-n-|\alpha|} \quad \text { by Hölder's inequality. }
\end{aligned}
$$

b) The proof of (18) is almost analogous to the above reasoning with the obvious simplification: In the numerator $\frac{1}{|y-z|^{n+1}}$ has to be replaced by $\frac{1}{|y-z|^{n}}$. Only the case $|\alpha|=2 m-n$ and $n$ even is different and will be carried out here.

The case: $|\alpha|=2 m-n$ and $n$ even.

We use Lemma A.2.

$$
\begin{aligned}
& \frac{\left|D_{z}^{\alpha} G_{m, n}(x, z)\right| L_{m, n}(z, y)}{L_{m, n}(x, y)} \\
& \preceq \frac{\log \left(2+\frac{d(x)}{|x-z|}\right) \min \left\{1, \frac{d(x)}{|x-z|}\right\}^{m} \min \left\{1, \frac{d(z)}{|x-z|}\right\}^{\max \{m-|\alpha|, 0\}} \frac{d(z)^{m}}{|z-y|^{n}}}{\frac{d(x)^{m}}{|x-y|^{n}}} \\
& \preceq \log \left(\frac{3}{|x-z|}\right) d(x)^{-m} d(z)^{m}|y-z|^{-n}\left(|x-z|^{n}+|y-z|^{n}\right) \\
& \cdot \min \left\{1, \frac{d(x)}{|x-z|}\right\}^{m} \min \left\{1, \frac{d(z)}{|x-z|}\right\}^{\max \{m-|\alpha|, 0\}} \\
& \preceq \log \left(\frac{3}{|x-z|}\right) d(x)^{-m} d(z)^{m}|y-z|^{-n}|x-z|^{n} \\
& \cdot\left(\frac{d(x)}{|x-z|}\right)^{\min \{m, n\}}\left(\frac{d(x)}{d(z)}\right)^{\max \{m-n, 0\}}\left(\frac{d(z)}{|x-z|}\right)^{\max \{m-|\alpha|, 0\}} \\
& +\log \left(\frac{3}{|x-z|}\right) d(x)^{-m} d(z)^{m}\left(\frac{d(x)}{d(z)}\right)^{m} \\
& \preceq \log \left(\frac{3}{|x-z|}\right) d(z)^{n}|y-z|^{-n}+\log \left(\frac{3}{|x-z|}\right) \preceq \log \left(\frac{3}{|x-z|}\right) .
\end{aligned}
$$


The estimates (17) and (18) in the Lemma above are integrable with respect to $z \in B$ uniformly in $x \in B, y \in \partial B$, if $|\alpha| \leq 2 m-2$. Our main result is a direct consequence of this fact.

Theorem 3.5. Let $s>\frac{1}{2}(n-2-m)(m-1)$. Then there exists $\varepsilon_{0}=\varepsilon_{0}(m, n, s)>0$ such that the following holds. with

If $\left\|b_{\alpha}\right\|_{C^{|\alpha|}(\bar{B})} \leq \varepsilon_{0}$ for $|\alpha| \leq 2 m-2$, then for every $\varphi \in C^{0}(\partial B), \psi \in C^{1}(\partial B)$

$$
\left.\begin{array}{l}
\psi \geq 0 \\
\varphi \geq s \psi
\end{array}\right\} \text { on } \partial B, \psi \neq \equiv 0 \text { or } \varphi \not \equiv 0
$$

the Dirichlet problem

$$
\begin{cases}(-\Delta)^{m} u+\sum_{|\alpha| \leq 2 m-2} b_{\alpha}(x) D^{\alpha} u=0 & \text { in } B \\ \left(-\frac{\partial}{\partial \nu}\right)^{j} u=0 & \text { on } \partial B \text { for } j=0, \ldots, m-3 \\ \left(-\frac{\partial}{\partial \nu}\right)^{m-2} u=\psi & \text { on } \partial B \\ \left(-\frac{\partial}{\partial \nu}\right)^{m-1} u=\varphi & \text { on } \partial B\end{cases}
$$

has a strictly positive solution $u \in W_{\mathrm{loc}}^{2 m, p}(B) \cap C^{m-1}(\bar{B})$ ( $p>1$ arbitrary):

$$
u>0 \text { in } B \text {. }
$$

Proof. For existence and regularity we refer to $[\mathrm{ADN}]$ and $[\mathrm{Ag}]$. First we assume additionally that $\psi \in C^{m+2, \gamma}(\partial B), \varphi \in C^{m+1, \gamma}(\partial B)$. We denote $\hat{\varphi}_{s}=\varphi-s \psi$ and we let $p>1$ be arbitrary. The operator

$$
\mathcal{L}_{m, n} \hat{\varphi}_{s}(x):=\int_{\partial B} L_{m, n}(x, y) \hat{\varphi}_{s}(y) d \omega(y)
$$

$\operatorname{maps} \mathcal{L}_{m, n}: C^{m+1, \gamma}(\partial B) \rightarrow C^{2 m, \gamma}(\bar{B}) \hookrightarrow W^{2 m, p}(B)$,

$$
\hat{\mathcal{K}}_{m, n, s} \psi(x):=\int_{\partial B} \hat{K}_{m, n, s}(x, y) \psi(y) d \omega(y)
$$

maps $\hat{\mathcal{K}}_{m, n, s}: C^{m+2, \gamma}(\partial B) \rightarrow C^{2 m, \gamma}(\bar{B}) \hookrightarrow W^{2 m, p}(B)$, while the Green operator

$$
\mathcal{G}_{m, n} f(x):=\int_{B} G_{m, n}(x, y) f(y) d y
$$

maps $\mathcal{G}_{m, n}: L^{p}(B) \rightarrow W^{2 m, p}(B) \cap W_{0}^{m, p}(B)$, see $[\mathrm{ADN}]$. We write $\mathcal{A}:=$ $\sum_{|\alpha| \leq 2 m-2} b_{\alpha}(.) D^{\alpha}$. The solution of (19) is given by $u=-\mathcal{G}_{m, n} \mathcal{A} u+\hat{\mathcal{K}}_{m, n, s} \psi+$ 
$\mathcal{L}_{m, n} \hat{\varphi}_{s}$ or $\left(\mathcal{I}+\mathcal{G}_{m, n} \mathcal{A}\right) u=\hat{\mathcal{K}}_{m, n, s} \psi+\mathcal{L}_{m, n} \hat{\varphi}_{s}$. Here $\mathcal{I}+\mathcal{G}_{m, n} \mathcal{A}$ is a bounded linear operator in $W^{2 m, p}(B) \cap W_{0}^{m, p}(B)$, which for sufficiently small $\varepsilon_{0}$ is invertible. Hence

$$
\begin{aligned}
u= & \left(\mathcal{I}+\mathcal{G}_{m, n} \mathcal{A}\right)^{-1} \hat{\mathcal{K}}_{m, n, s} \psi+\left(\mathcal{I}+\mathcal{G}_{m, n} \mathcal{A}\right)^{-1} \mathcal{L}_{m, n} \hat{\varphi}_{s} \\
= & \hat{\mathcal{K}}_{m, n, s} \psi+\sum_{i=1}^{\infty}\left(-\mathcal{G}_{m, n} \mathcal{A}\right)^{i} \hat{\mathcal{K}}_{m, n, s} \psi \\
& +\mathcal{L}_{m, n} \hat{\varphi}_{s}+\sum_{i=1}^{\infty}\left(-\mathcal{G}_{m, n} \mathcal{A}\right)^{i} \mathcal{L}_{m, n} \hat{\varphi}_{s}
\end{aligned}
$$

We only show how to deal with the first series containing $\hat{\mathcal{K}}_{m, n, s}$, the second series containing $\mathcal{L}_{m, n}$ is treated in the same way with some obvious simplifications. For $i \geq 1$ we integrate by parts. As $\mathcal{A}$ is of order $\leq 2 m-2$ and $\hat{\mathcal{K}}_{m, n, s} \psi$ vanishes on $\partial B$ of order $m-2$ no additional boundary integrals arise. By means of Fubini-Tonelli we obtain for $x \in B$ :

$$
\begin{gathered}
\left(-\mathcal{G}_{m, n} \mathcal{A}\right)^{i} \hat{\mathcal{K}}_{m, n, s} \psi(x)=(-1)^{i} \int_{z_{1} \in B} G_{m, n}\left(x, z_{1}\right) \mathcal{A}_{z_{1}} \int_{z_{2} \in B} G_{m, n}\left(z_{1}, z_{2}\right) \ldots \\
\ldots \mathcal{A}_{z_{i-1}} \int_{z_{i} \in B} G_{m, n}\left(z_{i-1}, z_{i}\right) \\
\quad \mathcal{A}_{z_{i}} \int_{y \in \partial B} \hat{K}_{m, n, s}\left(z_{i}, y\right) \psi(y) d \omega(y) d z_{i} \ldots d z_{1} \\
=\quad(-1)^{i} \int_{z_{1} \in B}\left(\mathcal{A}_{z_{1}}^{*} G_{m, n}\left(x, z_{1}\right)\right) \int_{z_{2} \in B}\left(\mathcal{A}_{z_{2}}^{*} G_{m, n}\left(z_{1}, z_{2}\right)\right) \ldots \\
\quad \ldots \int_{z_{i} \in B}\left(\mathcal{A}_{z_{i}}^{*} G_{m, n}\left(z_{i-1}, z_{i}\right)\right) \int_{y \in \partial B} \hat{K}_{m, n, s}\left(z_{i}, y\right) \psi(y) d \omega(y) d z_{i} \ldots d z_{1} \\
=(-1)^{i} \int_{B} \ldots \int_{B} \int_{\partial B}\left(\mathcal{A}_{z_{1}}^{*} G_{m, n}\left(x, z_{1}\right)\right)\left(\mathcal{A}_{z_{2}}^{*} G_{m, n}\left(z_{1}, z_{2}\right)\right) \cdot \ldots \\
\quad \ldots \cdot\left(\mathcal{A}_{z_{i}}^{*} G_{m, n}\left(z_{i-1}, z_{i}\right)\right) \hat{K}_{m, n, s}\left(z_{i}, y\right) \psi(y) d \omega(y) d\left(z_{1}, \ldots, z_{i}\right) .
\end{gathered}
$$

Here $\mathcal{A}^{*} .=\sum_{|\alpha| \leq 2 m-2}(-1)^{|\alpha|} D^{\alpha}\left(b_{\alpha}\right.$. ) is the (formally) adjoint operator of the perturbation $\mathcal{A}$. By virtue of Lemma 3.4 we find:

$$
\begin{aligned}
\left|\left(-\mathcal{G}_{m, n} \mathcal{A}\right)^{i} \hat{\mathcal{K}}_{m, n, s} \psi(x)\right| \\
\leq \int_{\partial B} \int_{B} \cdots \int_{B} \hat{K}_{m, n, s}(x, y) \frac{\left|\mathcal{A}_{z_{1}}^{*} G_{m, n}\left(x, z_{1}\right)\right| \hat{K}_{m, n, s}\left(z_{1}, y\right)}{\hat{K}_{m, n, s}(x, y)} \\
\quad \cdot \frac{\left|\mathcal{A}_{z_{2}}^{*} G_{m, n}\left(z_{1}, z_{2}\right)\right| \hat{K}_{m, n, s}\left(z_{2}, y\right)}{\hat{K}_{m, n, s}\left(z_{1}, y\right)} \cdot \ldots
\end{aligned}
$$




$$
\begin{aligned}
& \ldots \cdot \frac{\left|\mathcal{A}_{z_{i}}^{*} G_{m, n}\left(z_{i-1}, z_{i}\right)\right| \hat{K}_{m, n, s}\left(z_{i}, y\right)}{\hat{K}_{m, n, s}\left(z_{i-1}, y\right)} \psi(y) d\left(z_{1}, \ldots, z_{i}\right) d \omega(y) \\
\leq & \left(C_{0} \varepsilon_{0}\right)^{i} \int_{\partial B} \hat{K}_{m, n, s}(x, y) \psi(y) d \omega(y)=\left(C_{0} \varepsilon_{0}\right)^{i}\left(\hat{\mathcal{K}}_{m, n, s} \psi\right)(x) .
\end{aligned}
$$

Analogously we have:

$$
\left|\left(-\mathcal{G}_{m, n} \mathcal{A}\right)^{i} \mathcal{L}_{m, n} \hat{\varphi}_{s}(x)\right| \leq\left(\hat{C}_{0} \varepsilon_{0}\right)^{i}\left(\mathcal{L}_{m, n} \hat{\varphi}_{s}\right)(x) .
$$

The constants $C_{0}=C_{0}(m, n, s), \hat{C}_{0}=\hat{C}_{0}(m, n)$ do not depend on $i$. If $\varepsilon_{0}=$ $\varepsilon_{0}(m, n, s)>0$ is chosen sufficiently small, we come up with

(20) $u \geq \frac{1}{C} \hat{\mathcal{K}}_{m, n, s} \psi+\frac{1}{C} \mathcal{L}_{m, n} \hat{\varphi}_{s}$.

The general case $\varphi \in C^{0}(\partial B), \psi \in C^{1}(\partial B)$ follows from (20) with help of approximation, the maximum estimates of $[\mathrm{Ag}]$ and local $L^{p}$-estimates $[\mathrm{ADN}]$.

Remark. The Dirichlet problem (7) may be equivalently reformulated, if $m$ is odd:

$(21) \begin{cases}(-\Delta)^{m} u=0 & \text { in } B, \\ D^{\alpha} u=0 & \text { on } \partial B \quad \text { for }|\alpha| \leq m-3, \\ -\frac{\partial}{\partial \nu} \Delta^{(m-3) / 2} u=\tilde{\psi} & \text { on } \partial B, \\ \Delta^{(m-1) / 2} u=\tilde{\varphi} & \text { on } \partial B ;\end{cases}$

and if $m$ is even:

(22) $\begin{cases}(-\Delta)^{m} u=0 & \text { in } B, \\ D^{\alpha} u=0 & \text { on } \partial B \quad \text { for }|\alpha| \leq m-3, \\ \Delta^{(m-2) / 2} u=\tilde{\psi} & \text { on } \partial B \\ -\frac{\partial}{\partial \nu} \Delta^{(m-2) / 2} u=\tilde{\varphi} & \text { on } \partial B .\end{cases}$

Comparing with (7) we have

$$
\begin{aligned}
& \tilde{\psi}=\psi \\
& \tilde{\varphi}= \begin{cases}\varphi-\frac{1}{2}(m-1)(n-1) \psi, & \text { if } m \text { odd } \\
\varphi-\frac{1}{2}(m-2)(n-1) \psi, & \text { if } m \text { even }\end{cases}
\end{aligned}
$$


With regard to Proposition 3.2 positive data

$$
\tilde{\psi} \geq 0, \quad \tilde{\varphi} \geq 0
$$

yield a positive solution $u \geq 0$, provided

(23) $\frac{1}{2}(m-1)(n-2-m) \leq \begin{cases}\frac{1}{2}(m-1)(n-1), & \text { if } m \text { odd } \\ \frac{1}{2}(m-2)(n-1), & \text { if } m \text { even. }\end{cases}$

For $m$ odd this condition is always satisfied. In case of $m$ even the condition (23) is equivalent to $n \leq m^{2}$. In the latter case a perturbation result like Theorem 3.5 requires the strict inequality $n<m^{2}$. Moreover, it is a straightforward exercise to find the analogues to Proposition 3.2 and Theorem 3.5 for the boundary value problems (21) and (22), resp.

\section{A Appendix}

For the reader's convenience we collect a technical lemma and the Green function estimates from [GS1].

Lemma A.1. On $B^{2}$ (for $x, y \in B$ ) we have with $p, q \geq 0$ fixed:

$$
\begin{aligned}
& \min \left\{1, \frac{d(y)}{|x-y|}\right\} \sim \min \left\{1, \frac{d(y)}{d(x)}, \frac{d(y)}{|x-y|}\right\}, \\
& \min \left\{1, \frac{d(x) d(y)}{|x-y|^{2}}\right\} \sim \min \left\{\frac{d(y)}{d(x)}, \frac{d(x)}{d(y)}, \frac{d(x) d(y)}{|x-y|^{2}}\right\}, \\
& \min \left\{1, \frac{d(x)^{p} d(y)^{q}}{|x-y|^{p+q}}\right\} \sim \min \left\{1, \frac{d(x)^{p}}{|x-y|^{p}}, \frac{d(y)^{q}}{|x-y|^{q}}, \frac{d(x)^{p} d(y)^{q}}{|x-y|^{p+q}}\right\}, \\
& \min \left\{1, \frac{d(x)^{p} d(y)^{q}}{|x-y|^{p+q}}\right\} \sim \min \left\{1, \frac{d(x)}{|x-y|}\right\}^{p} \min \left\{1, \frac{d(y)}{|x-y|}\right\}^{q} .
\end{aligned}
$$

On $B^{2}$ (for $x, y \in B$ ) we have with $p, q \geq 0$ and $p+q>0$ fixed:

$$
\log \left(1+\frac{d(x)^{p} d(y)^{q}}{|x-y|^{p+q}}\right) \sim \log \left(2+\frac{d(y)}{|x-y|}\right) \min \left\{1, \frac{d(x)^{p} d(y)^{q}}{|x-y|^{p+q}}\right\}
$$

Lemma A.2. Let $\alpha \in \mathbb{N}_{0}^{n}$. Then on $B^{2}$ we have the following.

a) For $|\alpha| \geq 2 m-n$ and $n$ odd, or, $|\alpha|>2 m-n$ and $n$ even: 
- if $|\alpha| \leq m$ then

$$
\left|D_{x}^{\alpha} G_{m, n}(x, y)\right| \preceq|x-y|^{2 m-n-|\alpha|} \min \left\{1, \frac{d(x)^{m-|\alpha|} d(y)^{m}}{|x-y|^{2 m-|\alpha|}}\right\} ;
$$

- if $|\alpha| \geq m$ then

$$
\left|D_{x}^{\alpha} G_{m, n}(x, y)\right| \preceq|x-y|^{2 m-n-|\alpha|} \min \left\{1, \frac{d(y)^{m}}{|x-y|^{m}}\right\} .
$$

b) For $|\alpha|=2 m-n$ and $n$ even:

- if $|\alpha| \leq m$ (that is $m \leq n$ ) then

$$
\begin{aligned}
& \left|D_{x}^{\alpha} G_{m, n}(x, y)\right| \preceq \\
& \quad \preceq \log \left(2+\frac{d(y)}{|x-y|}\right) \min \left\{1, \frac{d(x)^{m-|\alpha|} d(y)^{m}}{|x-y|^{2 m-|\alpha|}}\right\} ;
\end{aligned}
$$

- if $|\alpha| \geq m$ (that is $m \geq n)$ then

$$
\left|D_{x}^{\alpha} G_{m, n}(x, y)\right| \preceq \log \left(2+\frac{d(y)}{|x-y|}\right) \min \left\{1, \frac{d(y)^{m}}{|x-y|^{m}}\right\} .
$$

c) For $|\alpha| \leq 2 m-n$ and $n$ odd, or, $|\alpha|<2 m-n$ and $n$ even:

- if $|\alpha| \leq m-\frac{1}{2} n$ then

$$
\begin{aligned}
& \left|D_{x}^{\alpha} G_{m, n}(x, y)\right| \preceq \\
& \preceq d(x)^{m-\frac{1}{2} n-|\alpha|} d(y)^{m-\frac{1}{2} n} \min \left\{1, \frac{d(x)^{\frac{1}{2} n} d(y)^{\frac{1}{2} n}}{|x-y|^{n}}\right\} ; \\
& - \text { if } m-\frac{1}{2} n \leq|\alpha| \leq m \text { then } \\
& \left|D_{x}^{\alpha} G_{m, n}(x, y)\right| \preceq \\
& \preceq d(y)^{2 m-n-|\alpha|} \min \left\{1, \frac{d(x)^{m-|\alpha|} d(y)^{n-m+|\alpha|}}{|x-y|^{n}}\right\} ;
\end{aligned}
$$

- if $m \leq|\alpha|$ then

$$
\left|D_{x}^{\alpha} G_{m, n}(x, y)\right| \preceq d(y)^{2 m-n-|\alpha|} \min \left\{1, \frac{d(y)^{n-m+|\alpha|}}{|x-y|^{n-m+|\alpha|}}\right\} .
$$

In general the following estimate is weaker than Part c) of Lemma A.2 but still appropriate and more convenient for our purposes. 
Corollary A.3. For $|\alpha| \leq 2 m-n$ and $n$ odd, or, $|\alpha|<2 m-n$ and $n$ even we have:

$$
\left|D_{x}^{\alpha} G_{m, n}(x, y)\right| \preceq d(x)^{m-\frac{1}{2} n-|\alpha|} d(y)^{m-\frac{1}{2} n} \min \left\{1, \frac{d(x)^{\frac{1}{2} n} d(y)^{\frac{1}{2} n}}{|x-y|^{n}}\right\} .
$$

\section{References}

[Ag] Sh. Agmon, Maximum theorems for solutions of higher order elliptic equations, Bull. Amer. Math. Soc. 66 (1960), 77-80.

[ADN] Sh. Agmon, A. Douglis and L. Nirenberg, Estimates near the boundary for solutions of elliptic partial differential equations satisfying general boundary conditions. I, Commun. Pure Appl. Math. 12 (1959), 623-727.

[Bo1] T. Boggio, Sull'equilibrio delle piastre elastiche incastrate, Rend. Acc. Lincei 10 (1901), 197-205.

[Bo2] T. Boggio, Sulle funzioni di Green d'ordine m, Rend. Circ. Mat. Palermo 20 (1905), 97-135.

[Co] C. V. Coffman, On the structure of solutions to $\Delta^{2} u=\lambda u$ which satisfy the clamped plate conditions on a right angle, SIAM J. Math. Anal. 13 (1982), 746757.

[CD] C. V. Coffman and R. J. Duffin, On the structure of biharmonic functions satisfying the clamped plate conditions on a right angle, Adv. Appl. Math. 1 (1980), 373-389.

[Du] R. J. Duffin, On a question of Hadamard concerning super-biharmonic functions, J. Math. Phys. 27 (1949), 253-258.

[Ed] J. Edenhofer, Eine Integraldarstellung der Lösung der Dirichletschen Aufgabe bei der Polypotentialgleichung im Falle einer Hyperkugel, Math. Nachr. 69 (1975), 149-162.

[Ga] P. R. Garabedian, A partial differential equation arising in conformal mapping, Pacific J. Math. 1 (1951), 485-524.

[GS1] H.-Ch. Grunau and G. Sweers, Positivity for equations involving polyharmonic operators with Dirichlet boundary conditions, Math. Ann. 307 (1997), 589-626.

[GS2] H.-Ch. Grunau and G. Sweers, Positivity for perturbations of polyharmonic operators with Dirichlet boundary conditions in two dimensions, Math. Nachr. 179 (1996), 89-102.

[GS3] H.-Ch. Grunau and G. Sweers, Positivity properties of elliptic boundary value problems of higher order, Proc. Second World Congress of Nonlinear Analysts, to appear. 
[Ha] J. Hadamard, Mémoire sur le problème d'analyse relatif à l'équilibre des plaques élastiques encastrées, in: Euvres de Jaques Hadamard, Tome II, pp. 515-641, Centre National de la Recherche Scientifique: Paris, 1968, Reprint of: Mémoires présentés par divers savants a l'Académie des Sciences 33 (1908), 1-128.

[He] P. J. H. Hedenmalm, A computation of Green functions for the weighted biharmonic operators $\Delta|z|^{-2 \alpha} \Delta$, with $\alpha>-1$, Duke Math. J. 75 (1994), 51-78.

$[\mathrm{KKM}]$ V. A. Kozlov, V. A. Kondrat'ev and V. G. Maz'ya, On sign variation and the absence of "strong" zeros of solutions of elliptic equations, Math. USSR Izvestiya 34 (1990), 337-353.

[Loe] Ch. Loewner, On generation of solutions of the biharmonic equation in the plane by conformal mappings, Pacific J. Math. 3 (1953), 417-436.

[Ni] M. Nicolesco, Les fonctions polyharmoniques, Hermann: Paris, 1936.

[Osh] St. Osher, On Green's function for the biharmonic equation in a right angle wedge, J. Math. Anal. Appl. 43 (1973), 705-716.

[ST] H. S. Shapiro and M. Tegmark, An elementary proof that the biharmonic Green function of an eccentric ellipse changes sign, SIAM Rev. 36 (1994), 99-101.

[So] R. Soranzo, A priori estimates and existence of positive solutions of a superlinear polyharmonic equation, Dyn. Syst. Appl. 3 (1994), 465-487.

[Sz] G. Szegö, Remark on the preceding paper of Charles Loewner, Pacific J. Math. 3 (1953), 437-446.

[Ta] G. Talenti, On the first eigenvalue of the clamped plate, in: H. Brezis, J. L. Lions (eds.), Nonlinear partial differential equations and their applications, Collège de France Seminar, Vol. VI, Pitman Research Notes Math. 109 (1984), 309-313. 